\title{
Spontaneous spin-up during the decay of 2D turbulence in a square container with rigid boundaries
}

\section{Citation for published version (APA):}

Clercx, H. J. H., Maassen, S. R., \& Heijst, van, G. J. F. (1998). Spontaneous spin-up during the decay of 2D turbulence in a square container with rigid boundaries. Physical Review Letters, 80(23), 5129-5132.

https://doi.org/10.1103/PhysRevLett.80.5129

DOI:

10.1103/PhysRevLett.80.5129

Document status and date:

Published: 01/01/1998

\section{Document Version:}

Publisher's PDF, also known as Version of Record (includes final page, issue and volume numbers)

\section{Please check the document version of this publication:}

- A submitted manuscript is the version of the article upon submission and before peer-review. There can be important differences between the submitted version and the official published version of record. People interested in the research are advised to contact the author for the final version of the publication, or visit the $\mathrm{DOI}$ to the publisher's website.

- The final author version and the galley proof are versions of the publication after peer review.

- The final published version features the final layout of the paper including the volume, issue and page numbers.

Link to publication

\section{General rights}

Copyright and moral rights for the publications made accessible in the public portal are retained by the authors and/or other copyright owners and it is a condition of accessing publications that users recognise and abide by the legal requirements associated with these rights.

- Users may download and print one copy of any publication from the public portal for the purpose of private study or research.

- You may not further distribute the material or use it for any profit-making activity or commercial gain

- You may freely distribute the URL identifying the publication in the public portal.

If the publication is distributed under the terms of Article 25fa of the Dutch Copyright Act, indicated by the "Taverne" license above, please follow below link for the End User Agreement:

www.tue.nl/taverne

Take down policy

If you believe that this document breaches copyright please contact us at:

openaccess@tue.nl

providing details and we will investigate your claim. 


\title{
Spontaneous Spin-Up during the Decay of 2D Turbulence in a Square Container with Rigid Boundaries
}

\author{
H. J. H. Clercx, S. R. Maassen, and G. J. F. van Heijst \\ Fluid Dynamics Laboratory, Department of Physics, Eindhoven University of Technology, P.O. Box 513, \\ 5600 MB, Eindhoven, The Netherlands
}

(Received 17 November 1997; revised manuscript received 7 April 1998)

\begin{abstract}
Direct numerical simulations of the decay of 2D turbulent Navier-Stokes flows inside a square container with no-slip boundaries are presented. Several numerical runs have been carried out for three different values of the Reynolds number. A surprising observation in a majority of these runs is the spontaneous spin-up of the flow during the initial stage of the self-organization of the flow, which is due to normal and shear stresses exerted on the fluid by the rigid boundaries. [S0031-9007(98)06284-X]
\end{abstract}

PACS numbers: 47.27.Eq, 47.32.Cc

Numerical studies of decaying two-dimensional (2D) turbulence, based on simulations of the Navier-Stokes equations for incompressible flows on a square domain with periodic boundary conditions, have revealed the emergence of coherent vortex structures such as monopoles, dipoles, and, occasionally, tripoles [1-4]. This process is commonly referred to as self-organization of the flow. By employing periodic boundary conditions it is implicitly assumed that boundaries have no significant effect on the decay process. We recall that selforganization of 2D turbulent flows contrasts strongly with the behavior of $3 \mathrm{D}$ turbulence. In the $3 \mathrm{D}$ case, energy cascades from the injection scale to the smallest scales where it is finally dissipated, even in the limit of vanishing viscosity. It might therefore be expected that at some distance from the boundaries 3D turbulence is almost unaffected by the presence of rigid walls. However, in 2D turbulence, due to self-organization of the flow, the average size of the vortices increases, and vortex-wall interactions will eventually play an important role in the decay of 2D turbulent flows on a bounded domain (with rigid walls). Additional arguments justifying a more fundamental study of the influence of boundaries on the flow dynamics are the following: Experiments with quasi-2D flows in rotating [5] or stratified fluids [6,7], or in electromagnetically forced flows in electrolyte solutions [8], are usually carried out in finite-sized containers with rigid boundaries. Furthermore, several theoretical approaches for investigating 2D flows take into account the presence of boundaries. Examples are the study by Pointin and Lundgren [9] on the statistical mechanics of 2D vortices in a bounded container and the classification study of self-organized structures in 2D turbulence in perfect fluids (Euler flows) by Chavanis and Sommeria [10]. The latter approach is based on an application of the maximum entropy theory as introduced by, e.g., Miller [11] and Robert and Sommeria [12].

Naturally arising questions concerning numerical and experimental studies of $2 \mathrm{D}$ decaying turbulence are the following: How is the decay scenario of 2D turbulence on a square domain modified by the presence of rigid boundaries? Is the decay scenario of 2D turbulence in containers with rigid boundaries consistent with predictions based on maximum entropy theories? Can the fluid spontaneously spin up by acquiring angular momentum due to the interaction of the flow with the boundaries? How anisotropic and inhomogeneous are the turbulent velocity fluctuations in the presence of boundaries? In this Letter, we focus only on questions concerning the largescale flow dynamics in decaying 2D turbulence.

Recently, Li et al. [13-15] reported results of computations of decaying 2D turbulence inside a circular rigid boundary. The simulations with no-slip boundaries and an initial velocity field containing a large amount of net angular momentum $L$ revealed a very slow decay of $L$. The angular momentum also appears to be a better constant of motion than the kinetic energy. For this case, the quasistationary intermediate state consists of a monopolar vortex in the center of the circular container. In contrast, runs with $L(t=0)=0$ showed the eventual formation of a rather persistent dipolar structure. The vorticity produced in the boundary layer between the dipole and the no-slip boundary is predominantly accumulated in the wake of the dipole.

We report here on results of several numerical simulations of decaying 2D turbulent flows on a square domain $(-1 \leq x \leq 1,-1 \leq y \leq 1)$ with impermeable boundaries for $\operatorname{Re}=1000,1500$, and 2000 with no-slip $(u=0$ and $v=0$, with $u$ and $v$ the components of the velocity in the $x$ and $y$ directions, respectively) boundary conditions. The Reynolds number of the flow is defined as $\operatorname{Re}=U W / \nu$, where $U$ is the rms velocity of the initial flow field, $W$ is the half-width of the container, and $\nu$ is the kinematic viscosity of the fluid. The numerical simulations of the 2D Navier-Stokes equations (without using hyperviscosity or any similar artificial dissipation on small or large scales) are performed with a 2D Chebyshev pseudospectral method, with a maximum of 289 Chebyshev modes in each direction. The time integration is based on the second order accurate semi-implicit Adams-Bashforth Crank-Nicolson scheme. The numerical calculations, except the evaluation of 
the nonlinear terms, are performed in spectral space. Fast-Fourier transform methods are used to evaluate the nonlinear terms following the procedure designed by Orszag [16], where the padding technique has been used for de-aliasing. Further details of the numerical procedure can be found in Ref. [17].

The initial condition for the velocity field, denoted by $\mathbf{u}_{i}$, is obtained by a zero-mean Gaussian random realization of the first $65 \times 65$ Chebyshev spectral coefficients of both $u_{i}$ and $v_{i}$, and subsequently applying a smoothing procedure in order to enforce $\mathbf{u}_{i}=0$ at the boundary of the domain. The variance $\sigma_{n m}$ of the velocity spectrum of $\mathbf{u}_{i}$ is chosen as

$$
\begin{gathered}
\sigma_{n m}^{2}=\frac{n}{\left\{1+[(1 / 8) n]^{4}\right\}} \frac{m}{\left\{1+[(1 / 8) m]^{4}\right\}}, \\
\text { with } 0 \leq n, m \leq 64,
\end{gathered}
$$

and $\sigma_{n m} \equiv 0$ for $n, m \geq 65$. The resulting flow field is denoted by $\mathbf{U}(x, y)$. The smoothing function is $f(x)=$ $\left\{1-\exp \left[-\beta\left(1-x^{2}\right)^{2}\right]\right\}$, with $\beta=100$. The initial velocity field is thus $\mathbf{u}_{i}(x, y)=f(x) f(y) \mathbf{U}(x, y)$, where the flow field is normalized in order to enforce the $L_{2}$ norm of the velocity per unit surface of the initial flow field to be equal to unity. It should be emphasized that for all numerical runs the kinetic energy of the flow field drops from $E(t=0)=2$ to $E\left(t=0^{+}\right) \cong 1$ during the first time integration step, because the initial velocity field, with $\nabla \cdot \mathbf{u}_{i} \neq 0$, is then projected onto the subspace of divergence-free velocity fields. A more proper treatment for obtaining a divergence-free initial velocity field is possible, but for the present simulations the procedure described above is sufficient; the details of the initial flow field were found to be not important.

The angular momentum of the flow, defined with respect to the center of the container, is

$$
\begin{aligned}
L & =\int_{-1}^{1} \int_{-1}^{1}[x v(x, y)-y u(x, y)] d x d y \\
& =-\frac{1}{2} \int_{-1}^{1} \int_{-1}^{1}\left(x^{2}+y^{2}\right) \omega(x, y) d x d y,
\end{aligned}
$$

where $\omega \equiv \partial v / \partial x-\partial u / \partial y$ represents the vorticity of the flow. Because of the initialization procedure of the flow field, the mean value of $L(t=0)$ over a large number of realizations is zero. However, for a particular realization the flow was given a net nonzero angular momentum, $L(t=0) \neq 0$, but still small compared to the angular momentum $L_{s b}$ of the same amount of fluid in solid

\section{no-slip, $\operatorname{Re}=2000$}

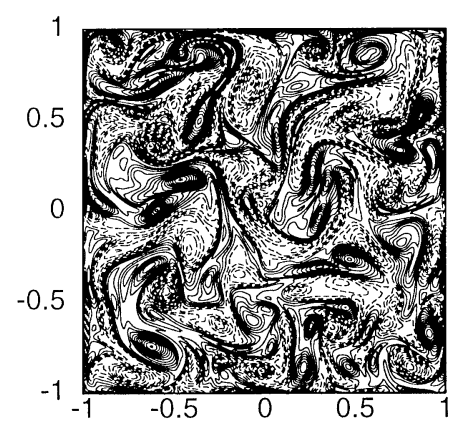

(a) $\mathrm{t}=\mathbf{1 . 0}$

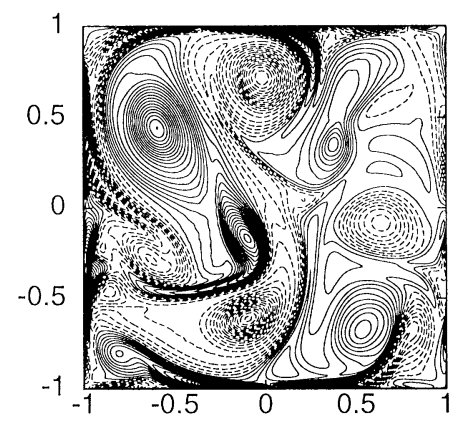

(d) $\mathbf{t}=\mathbf{1 0 . 0}$

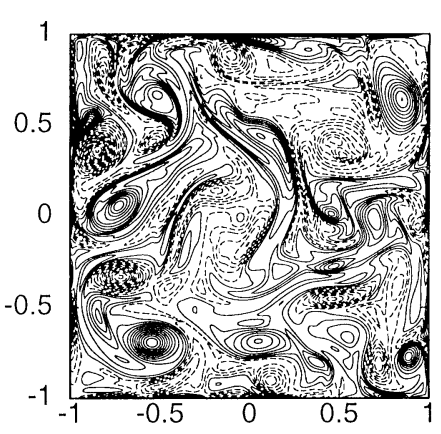

(b) $\mathbf{t}=\mathbf{2 . 0}$

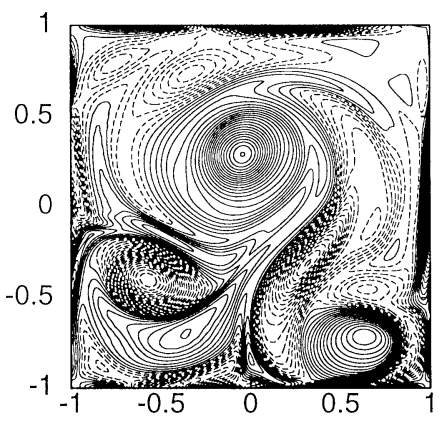

(e) $\mathbf{t}=\mathbf{2 0 . 0}$

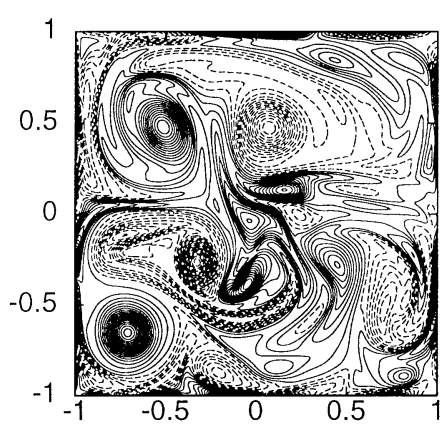

(c) $\mathbf{t}=5.0$

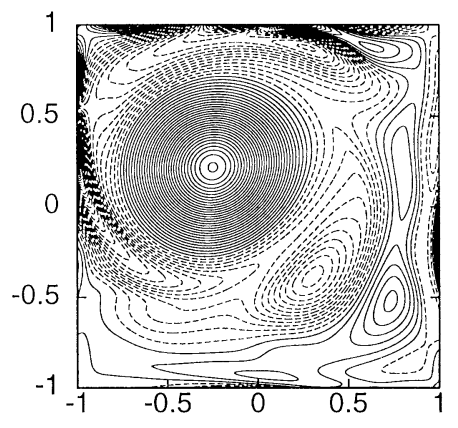

(f) $\mathbf{t}=\mathbf{5 0 . 0}$

FIG. 1. Vorticity contour plots of the simulation with no-slip boundary conditions, $\operatorname{Re}=2000$. Drawn contours ( positive vorticity, and dashed contours (_ _ - ) represent negative vorticity. The contour level increment is (a) and (b) 2, (c) 0.8 , (d) 0.5 , (e) 0.25 , (f) 0.1 . 
spontaneous spin-up for $\operatorname{Re}=\mathbf{2 0 0 0}$

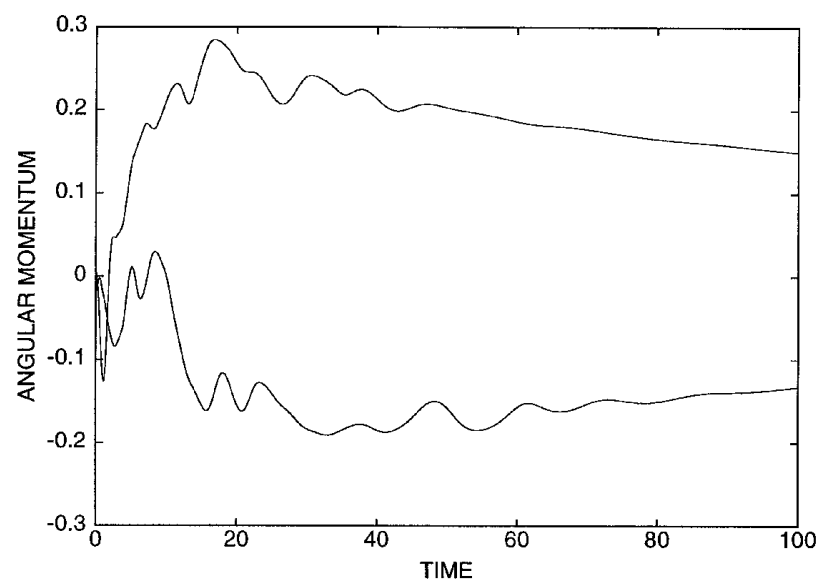

(a)

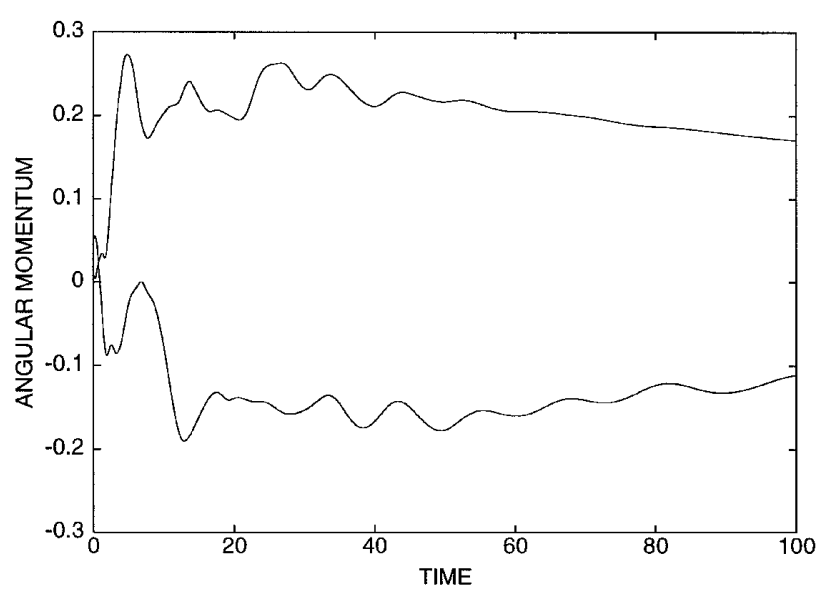

(b)

FIG. 2. The angular momentum $L$ plotted versus dimensionless time $t$ for $\operatorname{Re}=2000$ for four typical runs that show spontaneous spin-up.

body rotation and containing the same total kinetic energy $\left(E \cong 1\right.$ ), for which it can be shown that $\left|L_{s b}\right| \cong 2.3$.

Figure 1 shows some snapshots of decaying turbulence for $\operatorname{Re}=2000$ in a container with no-slip boundaries. Note that the total circulation for no-slip runs is always equal to zero (within machine accuracy). The no-slip runs predominantly show the appearance of intense vorticity filaments which arise from viscous vorticity production in the shear layers at the domain boundaries. These intense vorticity filaments are either injected into the flow interior or they roll up into a vorticity blob. In the latter case, they usually pair with the neighboring (primary) vortex, thus forming a dipolar structure. Figures 1(a) and 1(b) show the formation of intermediate-scale vortices. Vorticity-gradient sheets and several merging events are visible. A snapshot of vortex merging is even better observed in Fig. 1(d), which represents the flow evolution at $t=10$ (the time is made dimensionless with $W / U$, and is comparable with an eddy turnover time). For $t \geq 20$, the flow evolves to an intermediate state consisting of a monopole with a ring of oppositely signed vorticity.

As shown in Fig. 2(a) (upper curve), the absolute value of the angular momentum of the flow observed in the numerical run discussed in the previous paragraph increases suddenly from the value $|L| \cong 0$ to $|L| \cong 0.3$ during a short time interval $(5 \leq t \leq 15)$, and decays afterwards very slowly $(|L| \cong 0.15$ for $t=100)$. This sudden increase of the angular momentum reflects the spontaneous spin-up of the flow. Since the angular momentum of unbounded viscous flows is conserved when the total circulation (as in bounded domains with no-slip walls) is zero, the spontaneous spin-up is a process which is entirely due to the finiteness of the flow. This feature can also be observed from the rate of change of $L$, which can be expressed as a boundary integral containing contributions from viscous forces only [18]. A practical implication of the spin-up phenomenon is that a torque needs to be exerted on the container in order to prevent spontaneous rotation of the container since the total angular momentum of the torque-free fluid-container system is conserved. Note that the total linear impulse is zero for flows in domains with rigid walls at rest (the density of the fluid is assumed to be constant).

Spontaneous spin-up has been observed in several other runs. Three typical examples are shown in Figs. 2(a) and 2(b). Thirteen different runs have been carried out with $\operatorname{Re}=2000$ and eight of these runs showed spontaneous spin-up. Three runs show weak spin-up which is characterized by a rather strong increase of $|L|$ during the initial stage of the flow evolution, but a fast decrease of $|L|$ is observed for $t \geq 30$. Two runs show no spin-up at all. Typical properties of the flow observed in the eight runs that show spontaneous spin-up are the characteristic spin-up time, which is about $t \cong 17$, the spin-up amplitude, which is about $|L| \cong 0.22$, and the character of the flow during the intermediate stage of decay, which is always a strong monopolar or a rotating tripolar structure. Runs with a lower Reynolds number $(\operatorname{Re}=1500$ and 1000) show the same decay properties, although less pronounced for $\operatorname{Re}=1000$. Spontaneous spin-up in the simulations with $\operatorname{Re}=1500$ is always accompanied by the formation of a tripole or monopole as an intermediate state. The runs with $\mathrm{Re}=1000$ nearly always relax directly towards a rather strong monopole when spontaneous spin-up is observed (only in two cases a weak tripolar structure has been found). All data of the present runs are summarized in Table I.

During the decay of 2D turbulence, three major processes are relevant: turbulent spectral transfer due to nonlinear interactions, the selective decay mechanism, and in the later stage of the flow evolution (for $t \geq 150$ in the runs with $\operatorname{Re}=1500$ and 2000) the process of self-similar decay. In the latter process, the dynamics is strongly dominated by viscous decay (for a more elaborate discussion, see Ref. [19]). The three sets of simulations 
TABLE I. Several characteristics obtained from ensemble simulations of decaying 2D turbulence in square containers with no-slip boundaries. The characteristic spin-up times and amplitudes are based on averages of the runs showing spontaneous spin-up.

\begin{tabular}{lccc}
\hline \hline & $\mathrm{Re}=2000$ & $\mathrm{Re}=1500$ & $\mathrm{Re}=1000$ \\
\hline $\begin{array}{l}\text { Total number } \\
\quad \text { of runs }\end{array}$ & 13 & 12 & 12 \\
$\begin{array}{l}\text { Spontaneous } \\
\quad \text { spin-up }\end{array}$ & 8 & 8 & 7 \\
Weak spin-up & 3 & 2 & 1 \\
No spin-up & 2 & 2 & 4 \\
Spin-up time & 17 & 20 & 12 \\
Spin-up & & & \\
$\quad$ amplitude & 0.22 & 0.20 & 0.14 \\
\hline \hline
\end{tabular}

strongly indicate that the self-similarly decaying viscous mode with the lowest decay rate (a symmetric mode with a monopole in the center of the domain and a ring of oppositely signed vorticity near the boundaries) is rather dominant in the early decay stage and responsible for the spin-up phenomenon. In the minority of simulations that do not show any spin-up (20\% of all runs), the flow evolves to an antisymmetric intermediate state similar to, for example, a dipolar or a quadrupolar structure.

In summary, it can be concluded that numerical investigation of $2 \mathrm{D}$ decaying turbulence in square containers with rigid walls, based on simulations of the 2D NavierStokes equations, has revealed that the presence of rigid no-slip boundaries plays a crucial role in the flow evolution. A majority of the runs, performed at Reynolds numbers 2000, 1500, and 1000, show spontaneous spinup of the flow due to normal and shear stresses exerted on the fluid by the rigid boundaries. In the intermediate state of the runs that show spin-up the flow has a tripolar or monopolar character.

The authors gratefully acknowledge Professor David Montgomery for a number of useful discussions. One of us (S.R.M.) is grateful for support by the Netherlands Geosciences Foundation (GOA) with financial aid from the Netherlands Organization for Scientific Research (NWO).

[1] W. H. Matthaeus and D. Montgomery, Ann. N.Y. Acad. Sci. 357, 203 (1980).

[2] J. C. McWilliams, J. Fluid Mech. 146, 21 (1984).

[3] P. Santangelo, R. Benzi, and B. Legras, Phys. Fluids A 1, 1027 (1989).

[4] W. H. Matthaeus, W.T. Stribling, D. Martinez, S. Oughton, and D. Montgomery, Physica (Amsterdam) 51D, 531 (1991).

[5] E. J. Hopfinger and G. J.F. van Heijst, Annu. Rev. Fluid Mech. 25, 241 (1993).

[6] J. B. Flór, Ph.D. thesis, Eindhoven University of Technology, The Netherlands, 1994.

[7] B. M. Boubnov, S. B. Dalziel, and P.F. Linden, J. Fluid Mech. 261, 273 (1994).

[8] P. Tabeling, S. Burkhart, O. Cardoso, and H. Willaime, Phys. Rev. Lett. 67, 3772 (1991).

[9] Y.B. Pointin and T.S. Lundgren, Phys. Fluids 19, 1459 (1976).

[10] P. H. Chavanis and J. Sommeria, J. Fluid Mech. 314, 267 (1996).

[11] J. Miller, Phys. Rev. Lett. 65, 2137 (1990).

[12] R. Robert and J. Sommeria, J. Fluid Mech. 229, 291 (1991).

[13] S. Li and D. Montgomery, Phys. Lett. A 218, 281 (1996).

[14] S. Li, D. Montgomery, and W. B. Jones, J. Plasma Phys. 56, 615 (1996).

[15] S. Li, D. Montgomery, and W. B. Jones, Theor. Comput. Fluid Dyn. 9, 167 (1997).

[16] S. A. Orszag, Phys. Fluids Suppl. II 12, 250 (1969).

[17] H. J. H. Clercx, J. Comput. Phys. 137, 186 (1997).

[18] P. G. Saffman, Vortex Dynamics (Cambridge University Press, Cambridge, England, 1992).

[19] H. J. H. Clercx, S. R. Maassen, and G. J. F. van Heijst (to be published). 